\title{
Iliofemoral complications associated with thoracic endovascular aortic repair: Frequency, risk factors, and early and late outcomes
}

\author{
Frank C. Vandy, MD, ${ }^{\mathrm{a}}$ Micah Girotti, MD, ${ }^{\mathrm{b}}$ David M. Williams, MD,${ }^{\mathrm{c}}$ Jonathan L. Eliason, MD, ${ }^{\mathrm{a}}$ \\ Narasimham L. Dasika, MD, ${ }^{c}$ G. Michael Deeb, MD, ${ }^{b}$ and Himanshu J. Patel, MD ${ }^{\mathrm{b}}$
}

\begin{abstract}
Background: Risk factors and outcomes after iliofemoral complications after thoracic aortic endovascular repair remain poorly characterized. This study was performed to characterize factors influencing perioperative iliofemoral complications during thoracic aortic endovascular repair.
\end{abstract}

\begin{abstract}
Methods: All patients undergoing transfemoral thoracic aortic endovascular repair since 2005 with adequate preoperative aortoiliac 3-dimensional imaging $(n=126)$ were identified. Assessment of imaging was blinded with regard to occurrence of iliofemoral complications, defined as anything other than successful transfemoral device delivery and primary closure of an arteriotomy.
\end{abstract}

\begin{abstract}
Results: The complication rate was $12 \%(\mathrm{n}=15)$. Univariate analysis identified that female gender, preoperative ankle-brachial index, average and minimal iliac diameters, diameter difference between iliac artery and sheath size, and iliac morphology score (calculated by combining iliac tortuosity, calcification, and vessel diameter) were associated with iliofemoral complications (all $P<.05$ ). Multivariate analysis identified the $(1)$ difference between average iliac diameter and sheath size $(P=.014)$, (2) iliac artery morphology score $(P=.033)$, and (3) ankle-brachial index $(P=.012)$ as independent predictors for iliofemoral complications. Early mortality was higher in those with complications $(13.3 \%$ vs $1.8 \%, P=.069)$. Four-year freedom from limb loss, claudication, or revascularization was $97.9 \%$. Iliofemoral complications reduced late survival primarily as a result of increased mortality within the first year $(P=.047)$.
\end{abstract}

Conclusions: Thoracic aortic endovascular repair can be performed safely via a transfemoral approach. Alternative access in patients with high preoperative iliac artery morphology scores and device delivery size requirements over the native iliofemoral size may reduce iliofemoral complications. If early complications occur, prompt repair results in low rates of ischemic limb complications at late follow-up. (J Thorac Cardiovasc Surg 2014;147:960-5)

Despite the successful introduction of thoracic endovascular aortic repair (TEVAR) as a minimally invasive therapeutic option for the treatment of descending thoracic aortic aneurysms, it is associated with access and device delivery challenges. Passage of large-bore sheaths through diseased iliofemoral access vessels often precludes safe transfemoral TEVAR in up to $30 \%$ of patients. ${ }^{1}$ Although alternative access routes, including ascending aortic, iliac, and subclavian arteries, have been described, these options increase the morbidity, duration of hospitalization, and postoperative recovery of what is intended to be a minimally invasive approach to aneurysm repair. ${ }^{2,3}$ Modifications to the delivery catheters and sheaths (including tapered tips, hydrophilic coating, device diameter reduction, and

From the Departments of Surgery, ${ }^{\mathrm{a}}$ Cardiac Surgery, ${ }^{\mathrm{b}}$ and Radiology, ${ }^{\mathrm{c}}$ University of Michigan Cardiovascular Center, Ann Arbor, Mich.

Disclosures: Authors have nothing to disclose with regard to commercial support.

Received for publication Nov 11, 2012; accepted for publication Dec 20, 2012; available ahead of print March 18, 2013.

Address for reprints: Himanshu J. Patel, MD, Department of Cardiac Surgery, CVC Room 5144, 1500 E. Medical Center Drive SPC 5864, Ann Arbor, MI 48109-5864 (E-mail: hjpatel@med.umich.edu).

$0022-5223 / \$ 36.00$

Copyright (c) 2014 by The American Association for Thoracic Surgery

http://dx.doi.org/10.1016/j.jtcvs.2012.12.091 improved trackability) have helped overcome some aortoiliac anatomic limitations, but current series report a $9 \%$ to $22 \%$ incidence of access complications, thus contributing to perioperative morbidity in patients who are frequently elderly and debilitated. ${ }^{4-6}$

No previous study has fully evaluated these challenges with TEVAR, particularly with a rigorous 3-dimensional (3-D) analysis of preoperative imaging studies. With the advent of other minimally invasive cardiovascular therapies, including transcatheter aortic valve replacement, an examination of the incidence, risk factors for occurrence, and early and late outcomes of iliofemoral complications associated with large-bore diameter device delivery for TEVAR is timely and warranted.

\section{MATERIALS AND METHODS}

This single-center retrospective study was approved by the institutional review board of the University of Michigan Medical School (HUM 00053164). The primary outcome in this study was the incidence of iliofemoral access complications. The definition of an iliofemoral access complication was determined before beginning patient review and encompassed the following: (1) inability to successfully deliver the device into the aorta via a transfemoral approach; (2) rupture, dissection, tear, or thrombosis of the ipsilateral iliac artery or femoral artery, and (3) inability to achieve primary closure of the femoral artery. Secondary outcomes 


\section{Abbreviations and Acronyms}
ABI
$=$ ankle-brachial index
IMS = iliac morphology score
OR $=$ odds ratio
TEVAR $=$ thoracic endovascular aortic repair
3-D = 3-dimensional

included early mortality defined as in-house or 30-day death and freedom from limb loss, claudication, or revascularization.

All patients who underwent TEVAR from March 2005 to August 2011 were reviewed for study eligibility $(n=235)$. Before undergoing TEVAR, all patients underwent computed tomographic arteriography with 3-D reconstructions and modeling using M2S imaging software (M2S Inc, West Lebanon, $\mathrm{NH}$ ). Eighty patients were excluded from analysis on the basis of available imaging for the following reasons: (1) emergency status where time did not permit 3-D reconstruction, (2) 3-D models that did not include the iliofemoral vessels, and (3) missing M2S hard-copy compact discs with images no longer available for immediate review from the $\mathrm{M} 2 \mathrm{~S}$ server. Twenty-nine patients were excluded from the analysis because of planned delivery of the endograft via alternative access routes, including the iliac conduit or aortofemoral limb (18), ascending aorta (10), and carotid artery (1). The final study cohort consisted of 126 patients. Preoperative demographics and postoperative outcomes were collected retrospectively.

In 2002, a grading scale to objectively define the severity of anatomic factors in abdominal aortic aneurysms was developed by the ad hoc Committee for the Standardized Reporting Practices in Vascular Surgery of the Society for Vascular Surgery/American Association for Vascular Surgery. ${ }^{7}$ This scale designates a numeric value or score to the morphology of the aortic neck, aortic aneurysm, and iliac artery. Specifically, the iliac morphology score (IMS) includes extent of vessel calcification, vessel diameter or presence of occlusive disease, and vessel angulation or tortuosity. All available 3-D reconstructions were reviewed for the purpose of evaluating iliac anatomy and calculating this IMS.

The 3-D models were reviewed in a blinded fashion, such that the investigator did not know the operative and clinical outcome of the patient in question. Because the IMS was initially conceived for the purpose of TEVAR and included a component accounting for the landing zone of the iliac limbs, we developed a modified version using 3 components to evaluate the morphology of the iliac artery (Table 1). For the sake of simplicity, the common and external iliac arteries were evaluated as a continuous structure from the aortic bifurcation to the inguinal ligament, thus allowing calculation of a single IMS. A numeric value was assigned on the basis of varying degrees of calcification, artery diameter, and artery tortuosity. Diameter was recorded as minimal luminal and representative (average) diameter of the vessel. Tortuosity was calculated as the ratio between centerline luminal distance and straight line distance measured from the aortic bifurcation to the distal external artery at the inguinal ligament (Figure 1). The total score was calculated for the accessed side only, with a maximal score of 9 . To account for sheath oversizing, the outer diameter of the sheath used to deliver the endograft in each patient was recorded in millimeters. In patients who received more than 1 endograft, the largest sheath used was recorded. The difference between average iliac diameter and sheath outer diameter was recorded as sheath oversizing.

All TEVARs were performed in hybrid operating rooms under general anesthesia as previously described. Operative exposure of the femoral artery was performed via a 5-cm transverse infrainguinal incision. Percutaneous access was obtained in the contralateral femoral artery for placement of a $5 \mathrm{~F}$ sheath and a marked flush catheter. When intravascular ultrasound was used, an $8 \mathrm{~F}$ sheath was inserted. Ipsilateral femoral access was performed with a single wall puncture needle and a single Lunderquist wire (Cook Medical Inc, Bloomington, Ind) placed into the ascending aorta. Routine serial dilation of the ipsilateral iliofemoral vessel with hydrophilic dilators (Cook Medical Inc) up to a 24F size (or less if delivery sheath size was smaller) was performed before introducing the endograft. TAG (WL Gore \& Associates, Flagstaff, Ariz), TX2 (Cook Medical Inc), and Talent (Medtronic Inc, Minneapolis, Minn) endografts were used in this study. After deployment and removal of all devices, the common femoral artery was repaired with interrupted 5-0 polypropylene sutures, taking care to tack down all intimal flaps. Distal arterial signals and Doppler interrogation of the vessels were performed before closure of the wound. Ankle-brachial indices (ABIs) were obtained routinely on the first or second postoperative day. Completion imaging of the iliofemoral segment was performed selectively for altered pulse or Doppler signal identified after repairing the femoral artery.

\section{Statistical Analysis}

Statistical analysis was performed with SPSS (SPSS Inc, Chicago, Ill). Dichotomous variables were evaluated using chi-square analysis or Fisher exact test; continuous variables were evaluated using Student $t$ test. Multivariate models (binary logistic regression) were constructed using a forward conditional process to identify factors that were independently associated with each of the outcomes of interest. Factors used in multivariate analysis included those with a $P$ value of .1 or less on univariate analysis. Survival analysis was performed by Kaplan-Meier methods.

\section{RESULTS \\ Early Results}

The mean age for the entire cohort was 68.7 years with a slight predominance of male subjects $(54.8 \%)$. The demographics are listed in Table 2. Femoral artery exposure was made through a reoperative field in $12 \%$ of instances, and a mean of 1.9 stent grafts were used per patient. Indications for intervention included aneurysm $(60 \%)$, aortic dissection $(21 \%)$, blunt traumatic aortic injury $(17 \%)$, and penetrating ulcer $(2 \%)$.

In 126 patients, there were 15 iliofemoral access complications, yielding a complication rate of $12 \%$. In 8 patients, there was a rupture or dissection of the iliac artery; in 6 patients, the femoral artery required patch angioplasty to achieve closure. The endograft was unable to be delivered into the aorta via the transfemoral route in 1 patient. The presence of iliofemoral complications significantly increased postoperative length of stay (no complication median length of stay 5 days vs complication length of stay 6.5 days, $P=.007$ ).

By univariate analysis, those patients who sustained an iliofemoral complication were more likely to be female, to have smaller iliac arteries, and to have higher preoperative ABIs (all $P<.05$, Table 3 ). These patients also demonstrated more challenging anatomy as defined by a higher IMS (no complication IMS 2.7 vs complication IMS 3.7, $P<.001)$. Device delivery sheaths were, on average, more than $1 \mathrm{~mm}$ oversized in the complication cohort and slightly undersized in those patients who did not sustain a complication (Table 3). By multivariate analysis, independent predictors of iliofemoral access complications 
TABLE 1. Iliac artery morphology score (7)

\begin{tabular}{lcccc}
\hline \multicolumn{1}{c}{ Attribute } & Absent $=\mathbf{0}$ & Mild $=\mathbf{1}$ & Moderate $=\mathbf{2}$ & \multicolumn{1}{c}{ Severe $=\mathbf{3}$} \\
\hline Calcification & None & $<25 \%$ vessel length & $25 \%-50 \%$ vessel length & $>50 \%$ vessel length \\
Average diameter $(\mathrm{d})$ & $\mathrm{d}>10 \mathrm{~mm}$ & $\mathrm{~d}<8<10 \mathrm{~mm}$ & $\mathrm{~d}<7<8 \mathrm{~mm}$ & $\mathrm{~d}<7 \mathrm{~mm}$ \\
Iliac tortuosity index $(\tau)$ & $\tau<1.25$ & $\tau<1.25<1.5$ & $\tau<1.5<1.6$ & $\tau>1.6$ \\
\hline
\end{tabular}

included preoperative ABIs (odds ratio [OR], 19833; $P=.012)$, IMS (OR, $2.8 ; P=.033$ ), and sheath oversizing (mean iliac artery diameter minus sheath outer diameter OR, $0.38 ; P=.014$ ).

Early mortality, defined by in-hospital or 30-day death, was higher in those patients who had an iliofemoral complication $(13.3 \%$ vs $1.8 \%, P=.069)$. The only intraoperative death occurred in 1 patient who sustained an iliac artery rupture. This 76-year-old woman undergoing elective TEVAR for a $5.8-\mathrm{cm}$ descending aneurysm sustained a distal iliac artery rupture with resulting hypovolemic cardiac arrest during passage of the delivery sheath. Despite emergency iliac artery stent grafting with control, she was unable to

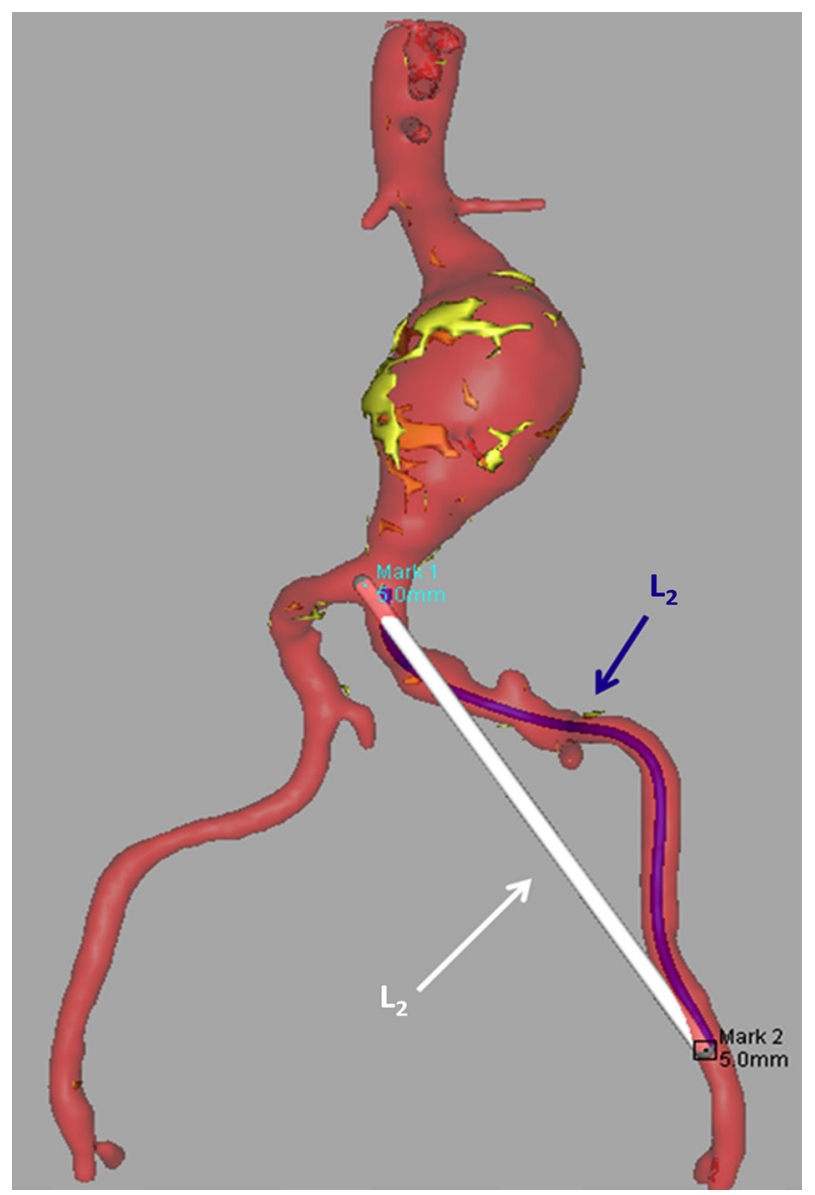

FIGURE 1. Assessment of iliac artery tortuosity index. The Society for Vascular Surgery iliac artery tortuosity index is determined by the ratio of the iliac artery length along the centerline of blood flow and the simple geometric straight line from the aortoiliac bifurcation to the femoral artery. be resuscitated and died. Her IMS was 5, including a score of 2 for calcification greater than $25 \%$ and 3 for a representative iliac diameter to sheath oversizing of $1.5 \mathrm{~mm}$. The only other early mortality in this study cohort was in a 71-year-old woman with severe chronic obstructive pulmonary disease undergoing TEVAR for an acute on chronic type B dissection with a 6-cm aneurysm. She required intraoperative left femoral patch angioplasty for reconstruction of her iliofemoral segment. Her death was due to development of permanent spinal cord ischemia with paralysis and subsequent failure to wean from the ventilator. She died on postoperative day 71 after comfort care measures were instituted.

TABLE 2. Demographics and comorbidities with univariate analysis

\begin{tabular}{|c|c|c|c|}
\hline Variable & $\begin{array}{c}\text { Iliofemoral } \\
\text { complication } \\
(\mathrm{N}=15) \\
\end{array}$ & $\begin{array}{c}\text { No } \\
\text { complication } \\
(\mathrm{N}=\mathbf{1 1 1}) \\
\end{array}$ & $\begin{array}{c}P \\
\text { value } \\
\end{array}$ \\
\hline \multicolumn{4}{|l|}{ Demographics and comorbidities } \\
\hline Age (y) & $73.2 \pm 10.6$ & $68.1 \pm 13.2$ & .15 \\
\hline Male sex & $3(20 \%)$ & $66(59.5 \%)$ & .005 \\
\hline Diabetes & $1(6.7 \%)$ & $17(15.3 \%)$ & .69 \\
\hline Coronary artery disease & $4(26.7 \%)$ & $45(40.5 \%)$ & .4 \\
\hline Prior CABG & $1(6.7 \%)$ & $18(16.2 \%)$ & .47 \\
\hline Prior PCI & $2(13.3 \%)$ & $16(14.4 \%)$ & 1.0 \\
\hline Congestive heart failure & $0(0 \%)$ & $10(9.1 \%)$ & .61 \\
\hline Carotid artery disease & $2(13.3 \%)$ & $18(16.2 \%)$ & 1.0 \\
\hline Hypertension & $13(86.7 \%)$ & $100(90.1 \%)$ & .66 \\
\hline Atrial fibrillation & $3(20 \%)$ & $16(14.4 \%)$ & .77 \\
\hline COPD & $4(26.7 \%)$ & $37(33.3 \%)$ & .77 \\
\hline History of tobacco abuse & $12(80 \%)$ & $81(73.0 \%)$ & .76 \\
\hline PVOD & $1(6.7 \%)$ & $16(14.4 \%)$ & .69 \\
\hline Preoperative ipsilateral ABI & $1.1 \pm 0.1$ & $1.0 \pm 0.2$ & .002 \\
\hline Prior AAA repair & $5(33.3 \%)$ & $31(27.9 \%)$ & .76 \\
\hline Preoperative creatinine $(\mathrm{mg} / \mathrm{dL})$ & $1.1 \pm 0.4$ & $1.2 \pm 1.1$ & .89 \\
\hline Dialysis-dependent renal failure & $0(0 \%)$ & $1(0.9 \%)$ & 1.0 \\
\hline \multicolumn{4}{|l|}{ Treated pathology } \\
\hline Aneurysm & $10(67 \%)$ & $65(58 \%)$ & .59 \\
\hline Penetrating ulcer & $1(6.7 \%)$ & $2(1.8 \%)$ & .78 \\
\hline Aortic dissection & $4(26.7 \%)$ & $23(20.7 \%)$ & .73 \\
\hline Blunt thoracic aortic injury & $0(0 \%)$ & $21(18.9 \%)$ & .32 \\
\hline Maximum aortic dimension $(\mathrm{cm})$ & $4.8 \pm 1.7$ & $4.6 \pm 1.7$ & .7 \\
\hline \multicolumn{4}{|l|}{ Procedural details } \\
\hline Reoperative groin exposure & $3(20 \%)$ & $12(10.8 \%)$ & .39 \\
\hline $\begin{array}{l}\text { No. of endografts used per } \\
\text { procedure }\end{array}$ & $2.2 \pm 0.8$ & $1.9 \pm 1.0$ & .22 \\
\hline
\end{tabular}

$\overline{C A B G \text {, Coronary artery bypass grafting; } P C I \text {, percutaneous coronary intervention; }}$ $C O P D$, chronic obstructive lung disease; $P V O D$, peripheral vascular occlusive disease; $A B I$, ankle-brachial index; $A A A$, abdominal aortic aneurysm. 
TABLE 3. Univariate analysis of risk factors associated with iliofemoral complications

\begin{tabular}{lccc}
\hline \multicolumn{1}{c}{ Variable } & $\begin{array}{c}\text { Iliofemoral } \\
\text { complication }\end{array}$ & $\begin{array}{c}\text { No } \\
\text { complication }\end{array}$ & $\boldsymbol{P}$ value \\
\hline Patients (n) & 15 & 111 & N/A \\
Female gender n (\%) & $12(80 \%)$ & $45(41 \%)$ & .009 \\
Age (y) & $74.7 \pm 10.6$ & $68.1 \pm 13.2$ & .16 \\
Iliac tortuosity index & $1.33 \pm 0.24$ & $1.22 \pm 0.15$ & .15 \\
Iliac calcium score & $1.15 \pm 0.53$ & $0.96 \pm 0.88$ & .48 \\
Average iliac diameter (mm) & $7.18 \pm 1.31$ & $8.64 \pm 1.99$ & .015 \\
Minimum iliac diameter (mm) & $6.16 \pm 1.0$ & $7.19 \pm 1.72$ & .041 \\
IMS & $3.77 \pm 0.72$ & $2.75 \pm 1.39$ & $<.001$ \\
Sheath oversizing (mm) & $-1.3 \pm 1.16$ & $0.12 \pm 1.94$ & $<.001$ \\
\hline N/A, Not available; $I M S$, iliac morphology score. & &
\end{tabular}

\section{Late Results}

Kaplan-Meier analysis suggested that the 4-year freedom from limb loss, claudication, or revascularization was $97.9 \% \pm 2.1 \%$ (Figure 2). One identified late event occurred in a man undergoing TEVAR for a $5.8-\mathrm{cm}$ saccular arch aneurysm. He sustained intraoperative external iliac artery avulsion and underwent successful emergency iliac artery stent graft repair. This limb thrombosed at 6 months and manifested as lifestyle-limiting claudication with a decrease in ABI from 1.01 to 0.43 . He underwent successful iliofemoral bypass with a Dacron graft at 3 years with resolution of symptoms. The only other late event occurred in

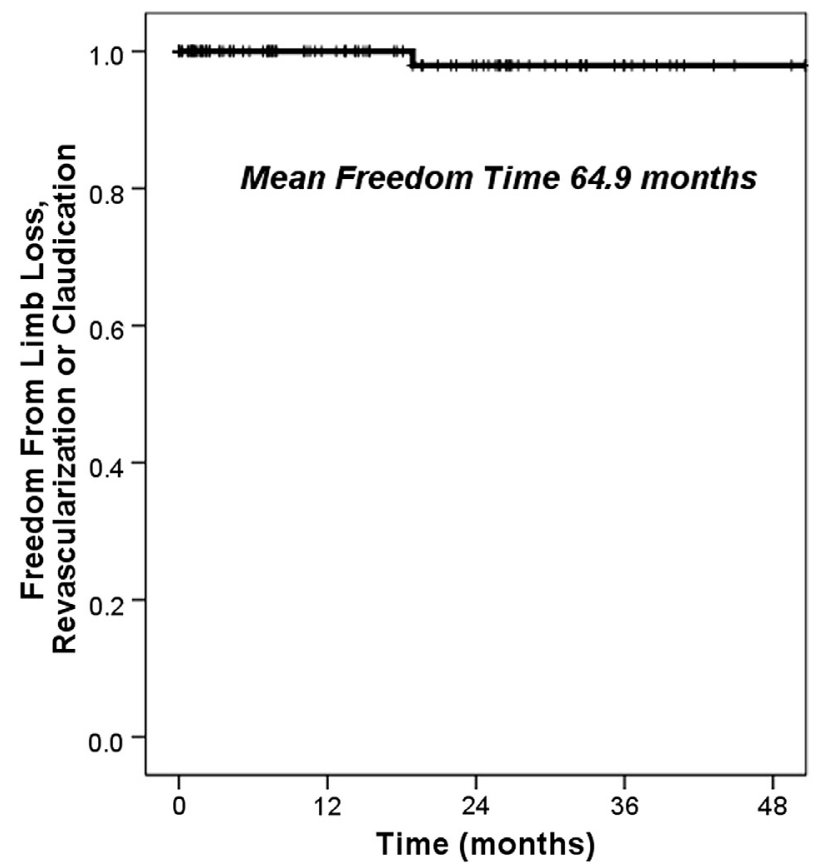

FIGURE 2. Freedom from late limb loss, claudication, or revascularization. This Kaplan-Meier survival analysis suggests that the freedom from limb loss, claudication, or revascularization at 4 years is $97.9 \% \pm 2.1 \%$.

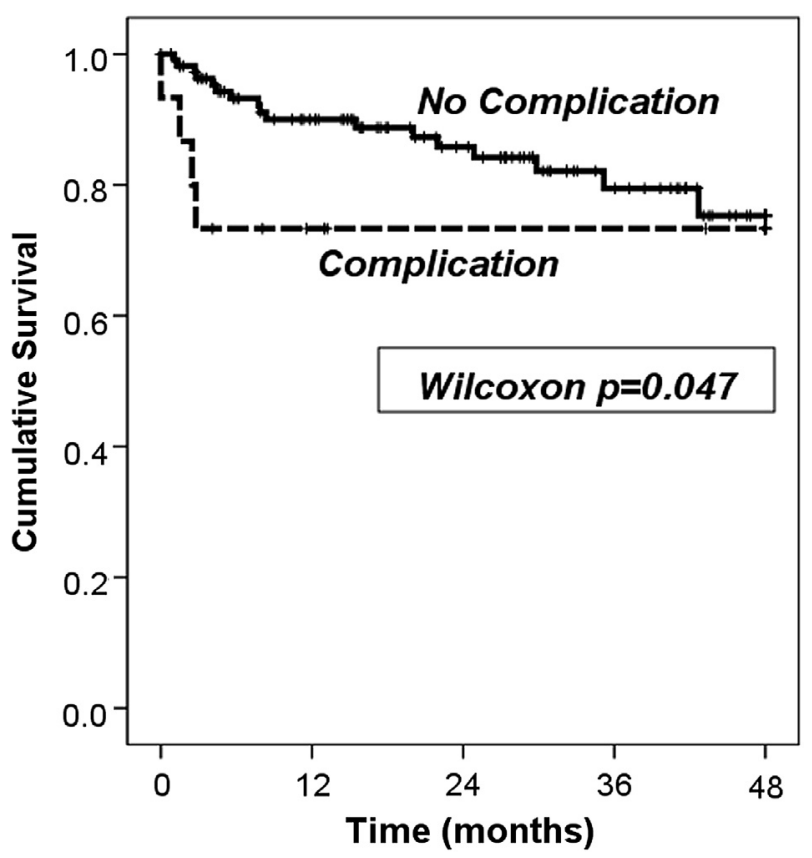

FIGURE 3. Late survival according to iliofemoral complications. The Kaplan-Meier survival analysis demonstrates that 4-year survival for the entire cohort is $76.0 \% \pm 5.3 \%$ (data not shown). When stratified by occurrence of perioperative iliofemoral complications, the 4-year survival is greater in those without complications than in those with complications (Wilcoxon $P=.047$ ). As the curves show, the predominant force of mortality is seen within the first year (no complication 1-year survival $90.0 \% \pm 3.0 \%$ vs complication 1-year survival $73.3 \% \pm 11.4 \%$ ).

a 70-year-old man who presented with multilevel claudication of the ipsilateral side approximately 5 years after TEVAR. He had developed intervening contralateral claudication requiring kissing balloon-expandable stents at the aortoiliac bifurcation 6 months before this symptom. He has responded well to maximum medical therapy, including cilostazol (Pletal; Otsuka America Pharmaceutical, Inc, Rockville, Md) therapy. There were no amputations in either subgroup.

When Kaplan-Meier analysis was performed for the entire cohort, the overall 4-year survival was $76.0 \% \pm 5.3 \%$. Most important, when a comparative analysis was performed, 4-year survival was significantly decreased in the complication subgroup (Wilcoxon $P=.046$ ). It is evident from the survival curve (Figure 3 ) that mortality in the complication subgroup predominantly occurs early after the operative procedure.

\section{DISCUSSION}

Endovascular approaches for a variety of thoracic aortic pathologies have expanded since the initial approval of the first thoracic endograft in $2005^{2,8}$ These minimally invasive treatment options have been successfully applied even in patients traditionally considered high risk or 
inoperable for conventional open aortic repair. Despite its less invasive appeal, TEVAR still has significant limitations. A recent study by Jackson and colleagues ${ }^{9}$ suggested significant anatomic constraints limiting the applicability of TEVAR in all patients evaluated for treatment. In their group of 126 patients evaluated for pivotal Food and Drug Administration-sponsored clinical trials of the Gore TAG and Medtronic Talent stent grafts, 33 patients were rejected on the basis of anatomic constraints. Although the overwhelming number of patients were rejected from inclusion into the trial on the basis of inadequate proximal or distal landing zones, 10 of the $33(30.3 \%)$ were rejected because of inadequate iliofemoral vessels. It should be noted that this trial permitted use of iliac artery conduits to aid in delivery, thus implying the rejection rate would have been substantially higher if only transfemoral delivery were considered. ${ }^{5}$

We have presented the largest series of patients undergoing TEVAR in which the IMS was shown to be significantly associated with an iliofemoral access complication. Although underreported and most likely underused, the IMS presents itself as a simple tool that should become part of every patient's preoperative planning to prevent delivery complications. These data suggest that in a patient with a score greater than 3, careful consideration should be given to alternative access. Certainly, it is relevant to consider how the total IMS was derived. A diminutive vessel with no angulation or calcification that scores a 3 is a different anatomic challenge than a large soft vessel that also scores a 3 because of severe tortuosity. Furthermore, the IMS score should be used in conjunction with other clinical factors, such as patient gender and size of intended device. This study demonstrated female gender to be a risk factor for complication, yet this is most likely attributable to small vessel diameter, because it is well known that women have smaller iliofemoral vessels than men. In addition, sheath oversizing was independently associated with a complication. As shown in Table 2, delivery of a sheath oversized by just $1 \mathrm{~mm}$ was associated with an iliofemoral complication. On the contrary, in the cohort that did not sustain a complication, the sheaths used were, on average, slightly smaller than the iliac artery.

This study also demonstrated that higher preoperative ABIs were independently associated with iliofemoral complications. This was an unexpected finding. On review of our database, we note that many patients in the complication cohort had ABIs exceeding 1.1. It is unclear whether this represents widespread arterial calcification. In this sense, ABIs elevated above normal range may be a surrogate marker for occlusive disease. Drawing conclusions from the ABI alone without knowledge of pulse volume recordings or cross-sectional lower-extremity imaging is limited.

The iliofemoral access complication rate $(12 \%)$ reported in this study was consistent with previous reports, even though 29 patients had been culled from the original cohort because of planned alternate access. ${ }^{5,6}$ Although some of these were planned in conjunction with additional procedures, such as TEVAR after arch debranching via median sternotomy, some of the exclusions resulted from preoperative judgment of inadequate iliofemoral access. In retrospect, an alternative delivery path such as a retroperitoneal iliac artery conduit would have been appropriate in even more of these patients. However, the decision to use a conduit to facilitate endograft delivery should be carefully considered in those patients with hostile iliac anatomy. The iliac conduit via retroperitoneal exposure has traditionally been the most common type of conduit used. However, as suggested by Lee and colleagues, ${ }^{10}$ this procedure is associated with increased morbidity. In their series of 164 patients undergoing an abdominal aortic endovascular repair, those patients who required an iliac conduit had a 2.6-fold increase in blood loss, $82 \%$ longer procedure time, 1.5 days additional hospital stay, and a 1.8-fold higher rate of perioperative complications. Similar results focusing just on patients undergoing TEVAR have been reported by Etezadi and colleagues. ${ }^{11}$ There was no significant difference in mortality in either study, which led the authors to conclude that although retroperitoneal procedures are significantly associated with longer hospital stay and more complications, such surgical procedures can significantly expand the number of patients who can undergo endovascular repair.

Given the operative morbidity associated with retroperitoneal conduits, other groups have described the lessinvasive internal endoconduit. ${ }^{3,12}$ In this procedure, a stent graft is deployed in the narrowed iliac segment, and an intentional rupture of the covered iliac segment is performed to allow safe delivery of a large-bore sheath. This technique must be used with caution in patients with innately small vessels. Furthermore, it may be necessary to exclude the hypogastric artery, which after TEVAR can contribute to an increased risk of spinal cord ischemia. ${ }^{13}$

The use of 3-D modeling is imperative not only to size the endograft but also to accurately derive the IMS. We routinely obtain 3-D vessel analysis on all patients undergoing elective or urgent TEVAR. Our current experience suggests that even in the setting of isolated thoracic aortic aneurysm disease, imaging of the aortoiliac and iliofemoral anatomy should be required before endovascular aortic repair. Centerline creation enables accurate measurements of seal zones, tortuosity, and C-arm correction angles. ${ }^{14}$

\section{Study Limitations}

The main limitations of this study include its retrospective nature and small sample size. Another limitation includes the lack of 3-D imaging availability in all the patients treated during this study period. However, it represents the only study to date to our knowledge that assesses 
the impact of iliofemoral complications after TEVAR on early and late outcomes.

\section{CONCLUSIONS}

Iliofemoral complication rates remain low after TEVAR. Although prompt recognition and repair of these complications allow a low rate of late limb ischemia, their occurrence portend a decrease in midterm survival. With the advent of newer endovascular treatments such as transcatheter aortic valve replacement, 3-D imaging including calculation of an IMS constitutes an important aspect of the preoperative evaluation to reduce iliofemoral access complications and allow a successful minimally invasive treatment.

\section{References}

1. Criado FJ, McKendrick C, Criado FR. Technical solutions for common problems in TEVAR: managing access and aortic branches. J Endovasc Ther. 2009; 16(Suppl 1):I63-79.

2. Brozzi NA, Roselli EE. Endovascular therapy for thoracic aortic aneurysms: state of the art in 2012. Curr Treat Options Cardiovasc Med. 2012;14:149-63.

3. Peterson BG, Matsumura JS. Creative options for large sheath access during aortic endografting. J Vasc Interv Radiol. 2008;19(6 Suppl):S22-6.

4. Rockman C. Reducing complications by better case selection: anatomic considerations. Semin Vasc Surg. 2004;17:298-306.
5. Garcia-Toca M, Eskandari MK. Regulatory TEVAR clinical trials. $J$ Vasc Surg. 2010;52(4 Suppl):22S-5S.

6. Fernandez JD, Craig JM, Garrett HE Jr, Burgar SR, Bush AJ. Endovascular management of iliac rupture during endovascular aneurysm repair. J Vasc Surg. 2009; 50:1293-300.

7. Chaikof EL, Fillinger MF, Matsumura JS, Rutherford RB, White GH, Blankensteijn JD, et al. Identifying and grading factors that modify the outcome of endovascular aortic aneurysm repair. J Vasc Surg. 2002;35:1061-6.

8. Makaroun MS, Dillavou ED, Kee ST, Sicard G, Chaikof E, Bavaria J, et al. Endovascular treatment of thoracic aortic aneurysms: results of the phase II multicenter trial of the GORE TAG thoracic endoprosthesis. J Vasc Surg. 2005;41:1-9.

9. Jackson BM, Carpenter JP, Fairman RM, Moser GW, Pochettino A, Woo EY, et al. Anatomic exclusion from endovascular repair of thoracic aortic aneurysm. J Vasc Surg. 2007;45:662-6.

10. Lee WA, Berceli SA, Huber TS, Ozaki CK, Flynn TC, Seeger JM. Morbidity with retroperitoneal procedures during endovascular abdominal aortic aneurysm repair. J Vasc Surg. 2003;38:459-65.

11. Etezadi V, Katzen BT, Benenati JF, Alehashemi S, Tsoukas AI, Puente OA Retroperitoneal versus direct femoral artery approach for thoracic endovascular aortic repair access: a case-control study. Ann Vasc Surg. 2011;25:340-4.

12. Peterson BG, Matsumura JS. Internal endoconduit: an innovative technique to address unfavorable iliac artery anatomy encountered during thoracic endovascular aortic repair. J Vasc Surg. 2008;47:441-5.

13. Feezor RJ, Lee WA. Strategies for detection and prevention of spinal cord ischemia during TEVAR. Semin Vasc Surg. 2009;22:187-92.

14. Bowman JN, Silverberg D, Ellozy S, Teodorescu V, Poblete H, Marin M, et al The role of anatomic factors in predicting success of endovascular repair of thoracic aortic aneurysms. Vasc Endovascular Surg. 2010;44:101-4. 